clinical specimens between January 2010-August 2015, of which 151 E. coli (24\%), 23 Klebsiella pneumoniae (3.7\%), 24 Enterobacter spp (3.8\%) and 5 Citrobacter spp (0.8\%), were resistant to extended spectrum cephalosporins (ESC) and exhibited an ESBL/AmpC phenotype when tested by the double disc synergy test (DDST) in combination with cefoxitin. Molecular characterisation of the 151 ESC resistant $E$. coli isolates, showed that 67 isolates (44\%) produced CTX-M type ESBLs, 55 isolates (36\%) carried blaCIT$\mathrm{M}$, an AmpC type beta-lactamase, whilst 12 isolates (7.9\%) carried both blaCTX-M and blaCIT-M. TEM and OXA-type beta-lactamases were also identified in 9.8\% and 3.5\% isolates, respectively. This study identified a high prevalence (24\%) of ESC resistant E. coli in clinical specimens from companion animals in the UK, which is much higher of that found in similar studies from pets in France $(3.7 \%)$ or Netherlands (2\%). The current study also characterises ESBL/AmpC producing E. coli isolates with focus on their genetic background, virulence factors and how these relate to clinical data and para-clinical information (cytology with evidence of Gram-negative bacteria, persistence of infection, evidence of inflammation) in order to determine their pathogenic potential. This continuing work seeks to establish the clinical significance of ESBL/AmpC producing $E$. coli isolates recovered from companion animal infections in order to support evidence-based decisions regarding treatment and infection control policies in veterinary settings.

\section{Health disorders of Pugs and Border Terriers}

\section{Elisabeth Darwent, Dave Brodbelt, David Church, Dan O'Neill}

Royal Veterinary College, London, UK

Health and welfare issues surrounding purebred dogs have been highly controversial since the BBC's Pedigree Dogs Exposed documentary in 2008, but several major reports have identified a deficiency of reliable disorder information within breeds. The limited data available mainly reflects referral or non-UK dog populations and is not necessarily representative of the wider population of UK dogs.

This study aimed to use clinical data from primary-care veterinary practices in the UK to report and compare the prevalence of common disorders in Border Terriers and Pugs.

Electronic patient records spanning 1st January to 31st December 2013 covering 1,327 Border Terriers and 1,015 Pugs within the VetCompass Programme were manually examined to extract data on all disorders diagnosed during this period. Border Terriers had a median age of 5.9 years (Interquartile range [IQR]: 3.1-9.2) and a median weight of 9.9kg (IQR: 8.6-11.5), compared with 3.0 years (IQR: 1.35.2) and $8.8 \mathrm{~kg}$ (IQR: 7.1-10.1) for Pugs. $47.2 \%$ of Border
Terriers were neutered, $34.1 \%$ were insured and $45.6 \%$ were microchipped, compared with 30.9\%, 25.5\% and 37.0\% for the Pugs. The most prevalent disorders of Border Terriers were periodontal disease $(17.6 \%, 95 \%$ confidence interval [Cl]: 15.6-19.6), obesity $(6.9 \%, 95 \% \mathrm{Cl}: 5.5-8.3)$ and otitis externa $(6.7 \%, 95 \% \mathrm{Cl}: 5.4-8.0)$. The most prevalent disorders of Pugs were obesity (13.1\%, 95\% Cl: 11.0-15.2), corneal disorders $(8.7 \%, 95 \% \mathrm{Cl}: 7.0-10.4)$ and otitis externa (7.5\%, 95\%Cl: 5.9-9.1). The head and neck was the most commonly affected body area in both breeds, but Pugs had a significantly higher prevalence of conditions affecting this region than Border Terriers (43.3\% vs 37.7\%, $\mathrm{p}=0.006$ ). Comparing the breeds for their top 24 disorders, Border Terriers had statistically significantly higher prevalence of four conditions; periodontal disease $(\mathrm{P}<0.001)$, conjunctivitis $(P=0.041)$, heart murmurs $(P<0.001)$ and cataracts $(P=0.001)$, whereas Pugs had statistically significantly higher prevalence in nine; obesity $(P<0.001)$, corneal disorders $(P<0.001)$, ear disorders $(\mathrm{P}<0.001)$, upper respiratory tract noise $(P<0.001)$, BOAS $(P<0.001)$, intertrigo $(P<0.001)$, retained deciduous teeth $(P<0.001)$, umbilical hernias $(P<0.001)$ and ocular discharge $(p=0.011)$.

These results provide much-needed health data on the general UK population of Pugs and Border Terriers and can assist with prioritisation of disorders for health reforms of these two breeds.

\section{Road Traffic Accidents (RTA) in dogs in the UK: epidemiology, clinical management and outcomes.}

\section{Georgina Harris', Dave Brodbelt', David Church', Karen Humm', Paul McGreevy², Peter Thomson'2, Dan O'Neill' \\ 1 The Royal Veterinary Collage, London, UK \\ 2 The University of Sydney, Sydney, Australia}

\section{OBJECTIVE}

To estimate the prevalence and risk factors for road traffic accidents (RTA) and describe the management and outcome of RTA events in dogs attending primary-care veterinary practices in the UK.

\section{METHOD}

Electronic patient records of dogs attending practices participating in the VetCompass Programme were assessed against selection criteria used to define RTA cases. The study population included 199,464 dogs attending 115 clinics across central and south-eastern England. The clinical notes were searched using a range of free-text search terms to identify potential RTA cases that were then manually verified to calculate prevalence. Univariable and multivariable logistic regression methods were used to evaluate associations between risk factors and RTA. 


\section{Oral presentations}

\section{RESULTS}

RTA prevalence was $0.41 \%$ (95\% confidence interval $(\mathrm{Cl})$ : 0.38-0.44). Of the RTA cases, 615 (74.9\%) were purebred, 322 (39.2\%) were female, and 285 (54.8\%) were insured. The median bodyweight was 17.0 (IQR: 9.1-26.9, range: 2.070.0) $\mathrm{kg}$ and the median age at diagnosis was 2.5 (IQR: 1.15.5, range: 0.0-17.0) years. After accounting for the effects of other factors using multivariable modelling, younger dogs had increased odds of an RTA event: dogs aged under three years showed 2.9 (95\% Cl 2.1-4.2, $\mathrm{P}<0.001)$ times the odds and dogs aged between six and nine years showed 1.8 (95\% $\mathrm{Cl} 1.2-2.7, \mathrm{P}=0.002)$ times the odds of an RTA event compared with dogs aged over 14 years. Males had $1.4(95 \% \mathrm{Cl}$ $1.2-1.6, P<0.001)$ times the odds of an RTA event compared with females. Overall, $22.9 \%$ of cases died from a cause related to their RTA event. Of dogs with information available, 34.0\% underwent diagnostic imaging, 29.4\% received intravenous fluid therapy, $71.1 \%$ received therapeutic pain relief, $46.0 \%$ were hospitalised and $15.6 \%$ had surgery performed under a general anaesthetic. Golden Retriever (OR: 0.2, 95\% Cl 0.1-0.7, P = 0.006), Shih-Tzu (OR: 0.5, 95\% Cl 0.2-0.9, P = 0.030) and West Highland White Terrier (OR: 0.4, 95\% Cl 0.2-0.8, P=0.015) showed reduced odds of RTA compared with crossbred dogs.

\section{CONCLUSION}

This study identified important breed effects and demographic factors that were associated with RTA in dogs, notably being young and male. This information can assist preventive action by highlighting the subsets of dogs most at risk.

\section{Breeder-reported canine} perinatal mortality in the United Kingdom in 2013 and 2014

\section{Samantha Scully ${ }^{1}$, Patrick Mitchell ${ }^{2}$, David Williams $^{3}$ \\ 1 Lincolnshire, UK \\ 2 Cambridgeshire, UK \\ 3 University of Cambridge, Cambridgeshire, UK}

This study aimed to determine current breeder-reported rates of canine perinatal mortality in the United Kingdom. For this study perinatal mortality was defined as the sum of stillbirths and neonate mortality, defined as the number of deaths from birth to 21 days of age.

An online survey was introduced to members of the Kennel Club Assured Breeders Scheme via email and social media. Breeders completed the survey anonymously, answering questions about one pedigree litter born during 2013 and 2014.

330 litters were reported representing 95 breeds and 2165 puppies. Perinatal mortality affected $47.6 \%$ of the litters. Excluding stillbirths, $27.9 \%$ of the litters suffered neonatal losses. Perinatal mortality at the puppy level was $16.5 \%$ with stillbirths accounting for $53 \%$ of these deaths. Of the puppies born alive, neonate mortality was $8.6 \%$ during the first 21 days with $8.5 \%$ being lost in the first week of life. Stillborn rates for bitches aged 1-2 years at parturition was 3\%, 2-3 years 34\%, 3-4 years 31\%, 4-5 years 17\%, 5-6 years $10 \%, 6-7$ years $4 \%$ and over 7 years of age $1 \%$.

Dogs were categorised into small breeds (less than $30 \mathrm{~cm}$ in height at the withers), medium $(31-51 \mathrm{~cm})$ and large (over $52 \mathrm{~cm}$ ). Perinatal mortality for the small breeds was $15.6 \%$, medium $13.3 \%$ and the large breeds $22.0 \%$. Stillbirth rates for these groups were, small $6.25 \%$, medium $8.21 \%$ and large $10.82 \%$. Neonatal mortality up to 21 days of age was $10.0 \%$ in the small breeds, $5.5 \%$ for the medium and $12.8 \%$ for large breeds.

This survey reports similar rates and patterns to previous studies in that most puppy deaths occur close to birth. Excluding stillbirths, neonatal losses are mainly in the first week of life although much higher in medium and large than in small breeds. The stillbirth rates for large breed dogs is double that for the small breeds. Bitches aged between 2-4 years have significantly higher rates of stillbirths.

It is notoriously difficult to gather sensitive information from breeders. This is the first time that social media and online anonymous data collection has been employed to collect puppy mortality information and may be a useful method to expand this data collection on a wider scale with a prospective cohort study. Examining husbandry and breeder experience may give some insight to explain the mortality patterns seen.

\section{Risk factors for road traffic accidents in the first year of a cat's life}

\section{Jessica Wilson, Timothy Gruffydd-Jones, Jane Murray}

University of Bristol, Bristol, UK

Road traffic accidents (RTAs) are a common cause of death and injury in domestic cats and a concern to many owners. This study assessed the potential risk factors for road traffic accidents in cats up to 12 months of age within a UK cat cohort known as 'The Bristol Cats Study'. Data was obtained from three questionnaires, completed by cat owners when their cats were 8-16 weeks old, 6 months old and 12 months old. Information was gathered regarding environmental conditions, cat characteristics and owner management factors. Univariable and multivariable logistic regression analysis was used to assess potential associations between these factors and RTAs. Cats were only included in the study if they had outdoor access and if their owners had completed the questionnaire for 12 month old cats. Cats were excluded if they had left the study prior to reaching 12 months of age for any reason other than an RTA, or if the owner was not sure whether or not their cat had been involved in an RTA. Of 1264 eligible study cats, 49 (3.9\%) had been involved in an RTA, of which $71.4 \%$ resulted in fatal injuries. Rural locations were 